\title{
Anticonvulsant Prophylaxis and Steroid Use in Adults With Metastatic Brain Tumors: ASCO and SNO Endorsement of the Congress of Neurological Surgeons Guidelines \\ ASSOCIATED \\ CONTENT
}

Appendix

Data Supplement

Author affiliations

and support

information (if

applicable) appear at the end of this article.

Accepted on November 30 , 2018 and published at jco.org on March 18, 2019: DOI https:// doi.org/10.1200/JCO. 18.02085

S.M.C. and M.A.V. were the Endorsement Panel co-chairs.

Editor's note:

This American Society of Clinical Oncology

Clinical Practice

Guideline provides recommendations, with comprehensive review and analyses of the relevant literature

for each

recommendation.

Additional information,

including a Data

Supplement with

additional evidence

tables, a Methodology

Supplement, slide

sets, clinical tools and

resources, and links to

patient information at

www.cancer.net, is

available at

www.asco.org/

neurooncology-

guidelines.

Clinical Practice

Guidelines

Committee

approval:

September 6, 2018

Reprint requests:

2318 Mill Road, Suite

800 , Alexandria, VA

22314;

guidelines@asco.org.

Susan M. Chang, MD; Hans Messersmith, MPH²; Manmeet Ahluwalia, MD; David Andrews, MD4; Priscilla K. Brastianos, MD';

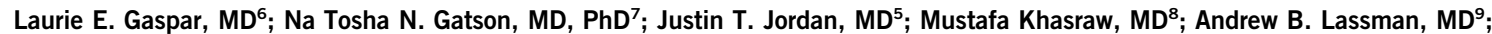
Julia Maues, MA ${ }^{10}$; Maciej Mrugala, MD, PhD ${ }^{11}$; Jeffrey Raizer, MD ${ }^{12}$; David Schiff, MD ${ }^{13}$; Glen Stevens, DO, PhD ${ }^{3}$; Ashley Sumrall, MD ${ }^{14}$; Martin van den Bent, $\mathrm{MD}^{15}$; and Michael $\mathrm{A}$. Vogelbaum, MD, $\mathrm{PhD}^{3}$

PURPOSE The Congress of Neurological Surgeons (CNS) has developed a series of guidelines for the treatment of adults with metastatic brain tumors, including systemic therapy and supportive care topics. ASCO has a policy and set of procedures for endorsing clinical practice guidelines that have been developed by other professional organizations.

METHODS Two CNS guidelines were reviewed for developmental rigor by methodologists, and an independent multidisciplinary Expert Panel was formed to review the content and assess agreement with the recommendations. The Expert Panel voted to endorse the two guidelines, and ASCO and Society for Neuro-Oncology (SNO) independently reviewed and approved the ASCO/SNO guideline endorsement.

RESULTS The ASCO/SNO Expert Panel determined that the recommendations from the CNS anticonvulsants and steroids guidelines, published January 9, 2019, are clear, thorough, and based on the most relevant scientific evidence. ASCO/SNO endorsed these two CNS guidelines with minor alterations.

RECOMMENDATIONS Key recommendations include the following: prophylactic antiepileptic drugs were not recommended for routine use; and corticosteroids, specifically dexamethasone, were recommended for temporary symptomatic relief in patients with neurologic symptoms and signs related to mass effect from brain metastases. Additional information is available at www.asco.org/neurooncology-guidelines.

J Clin Oncol 37:1130-1135. @ 2019 Society for Neuro-Oncology and the American Society of Clinical Oncology. This summary guideline endorsement was developed jointly by the Society for Neuro-Oncology \& the American Society of Clinical Oncology. All rights reserved.

\section{INTRODUCTION}

The Congress of Neurological Surgeons (CNS) has recently published a series of guidelines for the treatment and care of adults with metastatic brain tumors. ${ }^{1}$ Before publication, CNS requested that ASCO provide feedback on and consider endorsing the guideline series. As the care of the target population of these guidelines is an important issue for the members of both ASCO and the Society for Neuro-Oncology (SNO), ASCO and SNO conducted a joint guideline endorsement process for these guidelines.

\section{OVERVIEW OF THE ASCO GUIDELINE ENDORSEMENT PROCESS}

ASCO has policies and procedures for endorsing practice guidelines that have been developed by other professional organizations. The goal of guideline endorsement is to increase the number of high-quality, ASCO-vetted guidelines available to the ASCO membership. The ASCO endorsement process involves an assessment by ASCO staff of candidate guidelines for methodologic quality using the Rigor of Development subscale of the Appraisal of Guidelines for Research and Evaluation II (AGREE II) instrument. (See Methodology Supplement for more detail.)

\section{Disclaimer}

The clinical practice guidelines and other guidance published herein are provided by the American Society of Clinical Oncology, Inc. ("ASCO") to assist providers in clinical decision making. The information therein should not be relied upon as being complete or accurate, nor should it be considered as inclusive of all proper treatments or methods of care or as a statement of the standard of care. With the rapid development of scientific knowledge, new evidence may emerge between the time information is developed and when it is published or read. The information is not continually updated and may not reflect the most recent evidence. The information addresses only the topics specifically identified therein and is not applicable to other interventions, diseases, or stages of diseases. This information does not mandate any particular course of medical care. Further, the information is not intended to substitute for the independent professional 


\section{THE BOTTOM LINE}

\section{Anticonvulsant Prophylaxis and Steroid Use in Adults with Metastatic Brain Tumors: ASCO and SNO Endorsement of the Congress of Neurological Surgeons Guidelines}

ASCO and Society for Neuro-Oncology (SNO) endorse the Congress of Neurological Surgeons (CNS) Clinical Practice Guidelines, The Role of Prophylactic Anticonvulsants in the Treatment of Adults with Metastatic Brain Tumors and The Role of Steroids in the Treatment of Adults with Metastatic Brain Tumors, with some minor alterations.

\section{Guideline Questions}

\section{CNS Anticonvulsant Guideline}

Do prophylactic antiepileptic drugs decrease the risk of seizures in nonsurgical patients with brain metastases who are otherwise seizure free? Do prophylactic antiepileptic drugs decrease the risk of seizures in patients with brain metastases and no prior history of seizures in the postoperative setting?

\section{CNS Steroids Guideline}

Do steroids improve neurologic symptoms and/or quality of life in patients with metastatic brain tumors compared with supportive care only or other treatment options? If steroids are administered, what dose should be given?

\section{Target Population}

Adults with metastatic brain tumors.

\section{Target Audience}

Medical oncologists, neurologists, and others who provide care for adults with metastatic brain tumors.

\section{Methods}

An ASCO/SNO Expert Panel was convened to consider endorsing the CNS guideline recommendations that were based on a systematic review of the medical literature. The ASCO/SNO Expert Panel considered the methodology used in the CNS guidelines by considering the results from the Appraisal of Guidelines for Research and Evaluation II review instrument. The ASCO/SNO Expert Panel carefully reviewed the CNS guidelines content to determine its appropriateness for ASCO endorsement.

\section{Recommendations}

(Additions by the ASCO/SNO Expert Panel are in bold italics. See the note below regarding CNS recommendation levels.)

\section{CNS Anticonvulsants Guideline ${ }^{2}$}

- Level 3: Prophylactic antiepileptic drugs are not recommended for routine use in patients with brain metastases who did not undergo surgical resection and who are otherwise seizure free.

- Level 3: Routine postcraniotomy antiepileptic drug use for seizure-free patients with brain metastases is not recommended.

\section{CNS Steroids Guideline ${ }^{5}$ : Steroid Therapy Versus No Steroid Therapy}

Patients with asymptomatic brain metastases without mass effect:

- Insufficient evidence exists to make a treatment recommendation for this clinical scenario.

Patients with brain metastases with mild symptoms related to mass effect:

- Level 3: Corticosteroids are recommended to provide temporary symptomatic relief of symptoms related to increased intracranial pressure and edema secondary to brain metastases. It is recommended for patients who are symptomatic from metastatic disease to the brain that a starting dose of dexamethasone 4 to $8 \mathrm{mg} / \mathrm{d}$ be considered.

Patients with brain metastases with moderate to severe symptoms related to mass effect:

- Level 3: Corticosteroids are recommended to provide temporary symptomatic relief of symptoms related to increased intracranial pressure and edema secondary to brain metastases. If patients exhibit severe symptoms that are consistent with increased intracranial pressure, it is recommended that higher doses, such as $16 \mathrm{mg} / \mathrm{d}$ or more, be considered.

Choice of steroid:

- Level 3: If corticosteroids are administered, dexamethasone is the best drug choice given the available evidence.

$$
\text { (continued on following page) }
$$




\section{THE BOTTOM LINE (CONTINUED)}

Duration of corticosteroid administration:

- Level 3: Corticosteroids, if administered, should be tapered as rapidly as possible, but no faster than clinically tolerated, on the basis of an individualized treatment regimen and a full understanding of the long-term sequelae of corticosteroid therapy.

ASCO/SNO Expert Panel comment: The Panel's expert opinion is that, given the important adverse effects of steroids, the minimum effective dose (often no more than $4 \mathrm{mg}$ ) should be used where possible and night-time doses of steroids should be avoided to minimize toxicity.

Note regarding CNS Level 3 recommendation classification: CNS defines a Level 3 recommendation as one based on "Evidence from case series, comparative studies with historical controls, case reports, and expert opinion, as well as significantly flawed randomized controlled trials."

\section{Additional Resources}

More information, including a Data Supplement, a Methodology Supplement, slide sets, and clinical tools and resources, is available at www.asco.org/neurooncology-guidelines. Patient information is available at www.cancer.net

The CNS Guideline series can be found at https://www.cns.org/guidelines/guidelines-treatment-adults-metastaticbrain-tumors

ASCO believes that cancer clinical trials are vital to inform medical decisions and improve cancer care, and that all patients should have the opportunity to participate.

judgment of the treating provider, as the information does not account for individual variation among patients. Recommendations reflect high, moderate, or low confidence that the recommendation reflects the net effect of a given course of action. The use of words like "must," "must not," "should," and "should not" indicate that a course of action is recommended or not recommended for either most or many patients, but there is latitude for the treating physician to select other courses of action in individual cases. In all cases, the selected course of action should be considered by the treating provider in the context of treating the individual patient. Use of the information is voluntary. ASCO provides this information on an "as is" basis, and makes no warranty, express or implied, regarding the information. ASCO specifically disclaims any warranties of merchantability or fitness for a particular use or purpose. ASCO assumes no responsibility for any injury or damage to persons or property arising out of or related to any use of this information or for any errors or omissions.

\section{Guideline and Conflicts of Interest}

A multidisciplinary Expert Panel was assembled in accordance with ASCO's Conflict of Interest Policy Implementation for Clinical Practice Guidelines ("Policy," found at http://www.asco.org/rwc). All members of the Expert Panel completed ASCO's disclosure form, which requires disclosure of financial and other interests, including relationships with commercial entities that are reasonably likely to experience direct regulatory or commercial impact as a result of promulgation of the guideline. Categories for disclosure include employment; leadership; stock or other ownership; honoraria, consulting or advisory role; speaker's bureau; research funding; patents, royalties, other intellectual property; expert testimony; travel, accommodations, expenses; and other relationships. In accordance with the Policy, the majority of the members of the Expert Panel did not disclose any relationships constituting a conflict under the Policy.

\section{CLINICAL QUESTION(S) AND TARGET POPULATION}

CNS has published a series of eight guidelines ${ }^{1-9}$ that cover multiple aspects of the treatment of adults with metastatic brain tumors. Of these eight guidelines, four addressed systemic therapy and supportive care for these patients. Of the four guidelines that cover systemic therapy and supportive care, two were selected for endorsement-The Role of Prophylactic Anticonvulsants in the Treatment of Adults with Metastatic Brain Tumors ${ }^{2}$ and The Role of Steroids in the Treatment of Adults with Metastatic Brain Tumors. ${ }^{5}$ This work focuses on these two guidelines selected for endorsement. The two guidelines that were not endorsed reviewed the role of chemotherapy ${ }^{3}$ and various emerging therapies. ${ }^{4}$ Details on the newly identified evidence found and the review of the unendorsed guidelines can be found in Data Supplement 7. Because these two guidelines cover the same target population (adults with metastatic brain tumors) and because their recommendations have substantial interaction between the guidelines, ASCO conducted a common review of both guidelines. The complete set of clinical questions addressed by these two guidelines is provided in Supplemental Table 1. In summary, they address the role of anticonvulsant agents and steroids in the treatment of adults with brain metastases. 


\section{SUMMARY OF THE CNS GUIDELINE SERIES DEVELOPMENT METHODOLOGY}

The CNS guideline series was developed by a multidisciplinary author expert panel, the Metastatic Brain Tumor Guidelines Task Force, which included medical oncologists, radiation oncologists, neurologic surgeons, neurooncologists, and others. It built on a previous guideline series published in 2010. The literature search of MEDLINE, Embase, the Cochrane Database of Systemic Reviews, and the Cochrane Central Registry of Controlled Trials ended in December 2015 and began either in 2008 or 1990 depending on whether the specific guideline was an update of a 2010 guideline or new. Details of search strategies and study inclusion criteria and outcomes of interest are available in the guideline publications. ${ }^{1,2,5}$

The evidence review conducted by CNS identified many studies for inclusion in the guideline's qualitative synthesis of the literature, summarized in Data Supplement 4. Risk of bias and overall quality of the evidence, as well as the strength of the recommendations, were determined using CNS's published methods (https://www.cns.org/guidelines/ guideline-procedures-policies/guideline-developmentmethodology).

\section{RESULTS OF THE ASCO/SNO INITIAL METHODOLOGY AND CONTENT EVALUATION}

An initial methodology evaluation of the CNS guideline series was completed independently for each guideline in the series by two ASCO guideline staff members using the Rigor of Development subscale from the AGREE II instrument. Details of the scores are available in Data Supplement 6 . Each guideline in the series was also initially assessed by two content evaluators, members of the SNO guideline committee, who conducted a structured evaluation of the clinical content of the guideline series. Based on AGREE II and the clinical evaluations, the ASCO Clinical Practice Guideline Committee determined that the guideline series as a whole warranted detailed review by an ASCO/SNO Expert Panel (Appendix Table A1, online only) to determine which guidelines/recommendations could be endorsed.

This is the most recent information as of the publication date. For updates, the most recent information, and to submit new evidence, please visit www.asco.org/ neurooncology-guidelines.

\section{METHODS AND RESULTS OF THE ASCO UPDATED LITERATURE REVIEW}

This systematic review-based guideline product was developed by a multidisciplinary Expert Panel that included a patient representative and an ASCO guidelines staff member with health research methodology expertise. PubMed was searched from December 2015 to March 20, 2018. In addition, abstracts of the ASCO and American
Society for Radiation Oncology annual meetings from 2016 to 2017 were searched for relevant randomized controlled trials. The updated search was guided by the signals ${ }^{10}$ approach, which was designed to identify only new, potentially practice-changing data (signals) that might translate into revised practice recommendations. The approach relies on targeted routine literature searching and the expertise of ASCO/SNO Expert Panel members to help identify potential signals. As there would likely be some overlap among included articles, the search was structured to update the entire CNS guideline series literature search at the same time, with identified articles then assigned to the relevant guideline. The search was restricted to articles that were published in English. In addition, it was further restricted (compared with the original CNS search) to prospective studies of more than 30 participants and retrospective studies of more than 100 participants. The CNS guideline series used lower cutoffs which varied from guideline to guideline; however, as a result of concerns raised by the Expert Panel co-chairs that relevant studies may have been missed in the CNS guideline searches, an additional search was conducted back to January 2000 looking for studies with only "CNS," "central nervous system," or similar terms, but not the keyword "brain." The full search strategy and additional details can be found in Data Supplement 2. All funding for the administration of the project was provided by ASCO. The Methodology Supplement (available at www.asco.org/neurooncologyguidelines) provides additional information about the signals approach.

The updated search yielded 31 new articles. These articles are summarized in Data Supplement 1, Table 1. A review of these results found no new studies that were relevant to the anticonvulsants and steroids guidelines.

\section{RESULTS OF THE ASCO/SNO CONTENT REVIEW}

The ASCO/SNO Expert Panel reviewed the recommendations of the CNS guidelines in detail. Details of this review can be found in Data Supplement 4 and are summarized in this section.

\section{Anticonvulsants}

The Expert Panel found the recommendations reasonable, given the evidence, and hoped that although the guideline presents only negative recommendations it would still be useful in reducing the incidence of patients placed unnecessarily on anticonvulsant medications. The Expert Panel did decide to make a minor alteration to the first recommendation, adding the phrase, "for routine use," to make it consistent with the second recommendation. This guideline was endorsed.

\section{Steroids}

The Expert Panel agreed with the recommendations in this guideline. This guideline was endorsed. However, the Expert Panel felt that the recommendations in the guideline 
did not fully address the important adverse effects of steroid use, which can greatly affect a patient's quality of life, and added an additional comment encouraging the use of the minimal effective dose and avoiding night-time dosing. The Expert Panel also notes that a recent article by Arbor et al, ${ }^{11}$ published after the search conducted for this endorsement, suggests that higher doses of steroids may have negative consequences on outcomes in patients receiving programmed death ligand 1 inhibitors. This emerging literature should be monitored as it may affect the recommendations in future.

\section{ENDORSEMENT RECOMMENDATION}

ASCO and SNO endorse the CNS guidelines on anticonvulsants ${ }^{2}$ and steroids ${ }^{5}$ in the treatment of adults with brain metastases, with a minor alteration, as presented in the Bottom Line Box.

\section{RELATED ASCO GUIDELINES}

- Recommendations on Disease Management for Patients With Advanced Human Epidermal Growth Factor Receptor 2-Positive Breast Cancer and Brain Metastases: ASCO Clinical Practice Guideline Update (http://ascopubs.org/doi/ 10.1200/JCO.2018.79.2713)

\section{AFFILIATIONS}

${ }^{1}$ University of California, San Francisco, San Francisco, CA

${ }^{2}$ American Society of Clinical Oncology, Alexandria, VA

${ }^{3}$ Cleveland Clinic, Cleveland, $\mathrm{OH}$

${ }^{4}$ Thomas Jefferson University, Philadelphia, PA

${ }^{5}$ Massachusetts General Hospital, Boston, MA

${ }^{6}$ University of Colorado School of Medicine, Denver, CO

${ }^{7}$ Geisinger Neuroscience and Cancer Institutes, Danville, PA

${ }^{8}$ The University of Sydney, Sydney, NSW, Australia

${ }^{9}$ Columbia University Irving Medical Center, New York, NY

${ }^{10}$ Georgetown Breast Cancer Advocates, Washington, DC

${ }^{11}$ Mayo Clinic, Phoenix, AZ

${ }^{12}$ Northwestern University, Robert H Lurie Comprehensive Cancer Center of Northwestern University, Chicago, IL

${ }^{13}$ University of Virginia Medical Center, Charlottesville, VA

${ }^{14}$ Levine Cancer Institute, Charlotte, NC

${ }^{15}$ Erasmus MC Cancer Institute, Rotterdam, the Netherlands

\section{CORRESPONDING AUTHOR}

Hans Messersmith, MPH, American Society of Clinical Oncology, 2318

Mill Rd, Suite 800, Alexandria, VA 22314; e-mail: guidelines@asco.org.

\section{AUTHORS' DISCLOSURES OF POTENTIAL CONFLICTS OF INTEREST AND DATA AVAILABILITY STATEMENT \\ Disclosures provided by the authors and data availability statement (if applicable) are available with this article at DOI https://doi.org/10.1200/ JC0.18.02085.}

\section{AUTHOR CONTRIBUTIONS}

Conception and design: Susan M. Chang, Manmeet Ahluwalia, Mustafa Khasraw, Jeffrey Raizer, David Schiff, Glen Stevens, Martin van den Bent, Michael A. Vogelbaum

Administrative support: Susan M. Chang, Priscilla K. Brastianos Provision of study material or patients: Susan M. Chang

Collection and assembly of data: Manmeet Ahluwalia, Mustafa Khasraw, Maciej Mrugala, Jeffrey Raizer, David Schiff, Ashley Sumrall, Martin van den Bent

Data analysis and interpretation: Susan M. Chang, Hans Messersmith, Manmeet Ahluwalia, David Andrews, Priscilla K. Brastianos, Laurie E. Gaspar, Na Tosha N. Gatson, Justin T. Jordan, Mustafa Khasraw, Andrew B. Lassman, Julia Maues, Maciej Mrugala, Jeffrey Raizer, David Schiff, Glen Stevens, Martin van den Bent, Michael A. Vogelbaum

Manuscript writing: All authors

Final approval of manuscript: All authors

Accountable for all aspects of the work: All authors

\section{ACKNOWLEDGMENT}

The Expert Panel thanks Raetasha Dabney, MD, David Ollila, MD, and the Clinical Practice Guidelines Committee for thoughtful reviews and insightful comments on this guideline endorsement.

\section{REFERENCES}

1. Olson JJ, Kalkanis SN, Ryken TC: Congress of Neurological Surgeons systematic review and evidence-based guidelines for the treatment of adults with metastatic brain tumors: Executive summary. Neurosurgery doi:10.1093/neuros/nyy540 [epub ahead of print on January 9, 2019]

2. Chen CC, Rennert RC, Olson JJ: Congress of Neurological Surgeons systematic review and evidence-based guidelines on the role of prophylactic anticonvulsants in the treatment of adults with metastatic brain tumors. Neurosurgery doi:10.1093/neuros/nyy545 [epub ahead of print on January 9, 2019]

3. Sherman JH, Simon SL, Harrod T, et al: Congress of Neurological Surgeons systematic review and evidence-based guidelines on the role of chemotherapy in the management of adults with newly diagnosed metastatic brain tumors. Neurosurgery doi:10.1093/neuros/nyy544 [epub ahead of print on January 9, 2019]

4. Elder JB, Nahed BV, Linskey ME, et al: Congress of Neurological Surgeons systematic review and evidence-based guidelines on the role of emerging and investigational therapies for the treatment of adults with metastatic brain tumors. Neurosurgery doi:10.1093/neuros/nyy547 [epub ahead of print on January 9 , 2019]

5. Ryken TC, Kuo JS, Prabhu RS, et al: Congress of Neurological Surgeons systematic review and evidence-based guidelines on the role of steroids in the treatment of adults with metastatic brain tumors. Neurosurgery doi:10.1093/neuros/nyy546 [epub ahead of print on January 9, 2019]

6. Ammirati MA, Nahed BV, Andrews D, et al: Congress of Neurological Surgeons systematic review and evidence-based guidelines on treatment options for adults with multiple brain tumors. Neurosurgery doi:10.1093/neuros/nyy548 [epub ahead of print on January 9, 2019] 
7. Graber JJ, Cobbs CS, Olson JJ: Congress of Neurological Surgeons systematic review and evidence-based guidelines on the use of stereotactic radiosurgery in the treatment of adults with metastatic brain tumors. Neurosurgery doi:10.1093/neuros/nyy543 [epub ahead of print on January 9, 2019]

8. Nahed BV, Alvarez-Breckenridge C, Brastianos P, et al: Congress of Neurological Surgeons systematic review and evidence-based guidelines on the role of surgery in the management of adults with metastatic brain tumors. Neurosurgery doi:10.1093/neuros/nyy542 [epub ahead of print on January 9, 2019]

9. Gaspar LE, Prabhu RS, Hdeib A, et al: Congress of Neurological Surgeons systematic review and evidence-based guidelines on the role of whole brain radiation therapy in adults with newly diagnosed metastatic brain tumors. Neurosurgery doi:10.1093/neuros/nyy541 [epub ahead of print on January 9, 2019]

10. Shojania KG, Sampson M, Ansari MT, et al: How quickly do systematic reviews go out of date? A survival analysis. Ann Intern Med 147:224-233, 2007

11. Arbour KC, Mezquita L, Long N, et al: Impact of baseline steroids on efficacy of programmed cell death-1 and programmed death-ligand 1 blockade in patients with non-small-cell lung cancer. J Clin Oncol 36:2872-2878, 2018 
Anticonvulsant Prophylaxis and Steroid Use in Adults With Metastatic Brain Tumors: ASCO and SNO Endorsement of the Congress of Neurological Surgeons Guidelines

The following represents disclosure information provided by authors of this manuscript. All relationships are considered compensated. Relationships are self-held unless noted. I = Immediate Family Member, Inst = My Institution. Relationships may not relate to the subject matter of this manuscript. For more information about ASCO's conflict of interest policy, please refer to www.asco.org/rwc or ascopubs.org/jco/site/ifc.

Susan M. Chang

Consulting or Advisory Role: Tocagen

Research Funding: Novartis (Inst), Agios (Inst)

Manmeet Ahluwalia

Stock and Other Ownership Interests: MimiVax

Honoraria: Prime Oncology, Elsevier, Itamar Medical (I)

Consulting or Advisory Role: Monteris Medical, AstraZeneca, Bristol-Myers

Squibb, AbbVie, CBT Pharmaceuticals, Kadmon, VBI Vaccines

Research Funding: Novartis, TRACON Pharma, Novocure, Spectrum

Pharmaceuticals, Eli Lilly, ImClone, Boehringer Ingelheim, AstraZeneca

David Andrews

Employment: Imvax

Leadership: Imvax

Stock and Other Ownership Interests: Imvax

Consulting or Advisory Role: Imvax

Patents, Royalties, Other Intellectual Property: Thomas Jefferson University holds the patents and Imvax has the exclusive rights to the license

Priscilla K. Brastianos

Honoraria: Merck, Genentech, Angiochem

Consulting or Advisory Role: Genentech, Eli Lilly

Advisory Role: Angiochem

Research Funding: Merck, Pfizer

Laurie E. Gaspar

Honoraria: AstraZeneca

Consulting or Advisory Role: AstraZeneca

Other Relationship: American Association of Neurosurgery, National Cancer Institute Thoracic Malignancy Steering Committee

Justin T. Jordan

Patents, Royalties, Other Intellectual Property: Textbook royalties Other Relationship: Shepherd Therapeutics, Shepherd Foundation, NF Network

Mustafa Khasraw

Honoraria: Pfizer, Novartis

Consulting or Advisory Role: Bristol-Myers Squibb, AbbVie, Eli Lilly

Research Funding: AbbVie (Inst), Bristol-Myers Squibb (Inst), Specialised

Therapeutics (Inst)

Travel, Accommodations, Expenses: Genentech

Andrew B. Lassman

Honoraria: Prime Oncology, WebMD, American Society of Clinical Oncology, Italian Association for Cancer Research, Focus Forward Incentives, Research America, Gerson Lehrman Group, Guidepoint Global, Health Advisors Bureau, Medefield, MedSurvey, Olson Research Group, SAI MedPartners, Schlesinger Associates, Market Strategies International, Defined Health,

Marketplusresearch.com, AbbVie

Consulting or Advisory Role: BioClinica, AbbVie, Sapience Therapeutics, AstraZeneca, Novocure, Kadmon, Cortice, Celgene, Agios

Research Funding: AbbVie (Inst), Novartis (Inst), Karyopharm Therapeutics (Inst), Genentech (Inst), Novocure (Inst), Aeterna Zentaris (Inst), Pfizer (Inst), Bayer (Inst), Onyx Pharmaceuticals (Inst), Agenus (Inst), GlaxoSmithKline (Inst), Stemline Therapeutics (Inst), Northwest Biotherapeutics (Inst), Plexxikon (Inst), Tocagen (Inst), Regeneron (Inst), VBL Therapeutics (Inst), e-Therapeutics (Inst) Bristol-Myers Squibb (Inst), ImmunoCellular Therapeutics (Inst), Merck (Inst), Amgen (Inst), Celldex (Inst), Millennium Pharmaceuticals (Inst), Medlmmune (Inst), Boehringer Ingelheim (Inst), Kadmon (Inst), RTOG Foundation (Inst), Boston Biomedical (Inst), BeiGene (Inst), Diffusion Pharmaceuticals (Inst), Agios (Inst), Celgene (Inst), Vascular Biogenics (Inst), Angiochem (Inst), Northwest Biotherapeutics (Inst), Orbus (Inst), VBI Vaccines (Inst)

Travel, Accommodations, Expenses: Karyopharm Therapeutics, Tocagen, Radiological Society of North America, AbbVie, Agios, Novocure, NRG Oncology Foundation, Celgene, Kadmon, Bristol-Myers Squibb

Other Relationship: Medical expert in malpractice/disability cases

\section{Maciej Mrugala}

Consulting or Advisory Role: Novocure, AbbVie

Research Funding: Arbor Pharmaceuticals

Travel, Accommodations, Expenses: AbbVie, Novocure

Jeffrey Raizer

Employment: Agenus, Celldex, Astellas Pharma

Stock and Other Ownership Interests: Agenus, Exicure, Cancer Action Now Celldex

Honoraria: ZIOPHARM Oncology

Consulting or Advisory Role: ZIOPHARM Oncology

Research Funding: Novartis, Genentech, Plexxikon, Merck, Stemline

Therapeutics, AstraZeneca, Medlmmune

David Schiff

Consulting or Advisory Role: Orbus Therapeutics, Monteris Medical, Cavion (Inst)

Research Funding: Bayer (Inst)

Ashley Sumrall

Stock and Other Ownership Interests: Novocure (I)

Honoraria: Gerson Lehrman Group, Cardinal Health

Consulting or Advisory Role: Novocure, Amgen, AbbVie, Cardinal Health, Merck Speakers' Bureau: Bristol-Myers Squibb, Novocure, Prime Oncology

Research Funding: Bristol-Myers Squibb (Inst), Novocure (Inst)

Travel, Accommodations, Expenses: Novocure, Bristol-Myers Squibb, Xcenda, American Association of Neurologic Surgeons

Martin van den Bent

Consulting or Advisory Role: AbbVie, Celldex, Bristol-Myers Squibb, MSD,

Daiichi Sankyo, Celgene, Agios

Research Funding: AbbVie (Inst)

Michael A. Vogelbaum

Stock and Other Ownership Interests: Infusion Therapeutics

Honoraria: Medicenna

Research Funding: Tocagen (Inst), DNAtrix (Inst), Medicenna (Inst), Infusion

Therapeutics (Inst)

Patents, Royalties, Other Intellectual Property: Patents for drug delivery

devices for the brain

Travel, Accommodations, Expenses: Infusion Therapeutics

No other potential conflicts of interest were reported. 


\section{APPENDIX}

TABLE A1. Anticonvulsant Prophylaxis and Steroid Use in Adults With Metastatic Brain Tumors: ASCO and Society for Neuro-Oncology Joint Endorsement of the Congress of Neurological Surgeons Guidelines Expert Panel Membership

Name

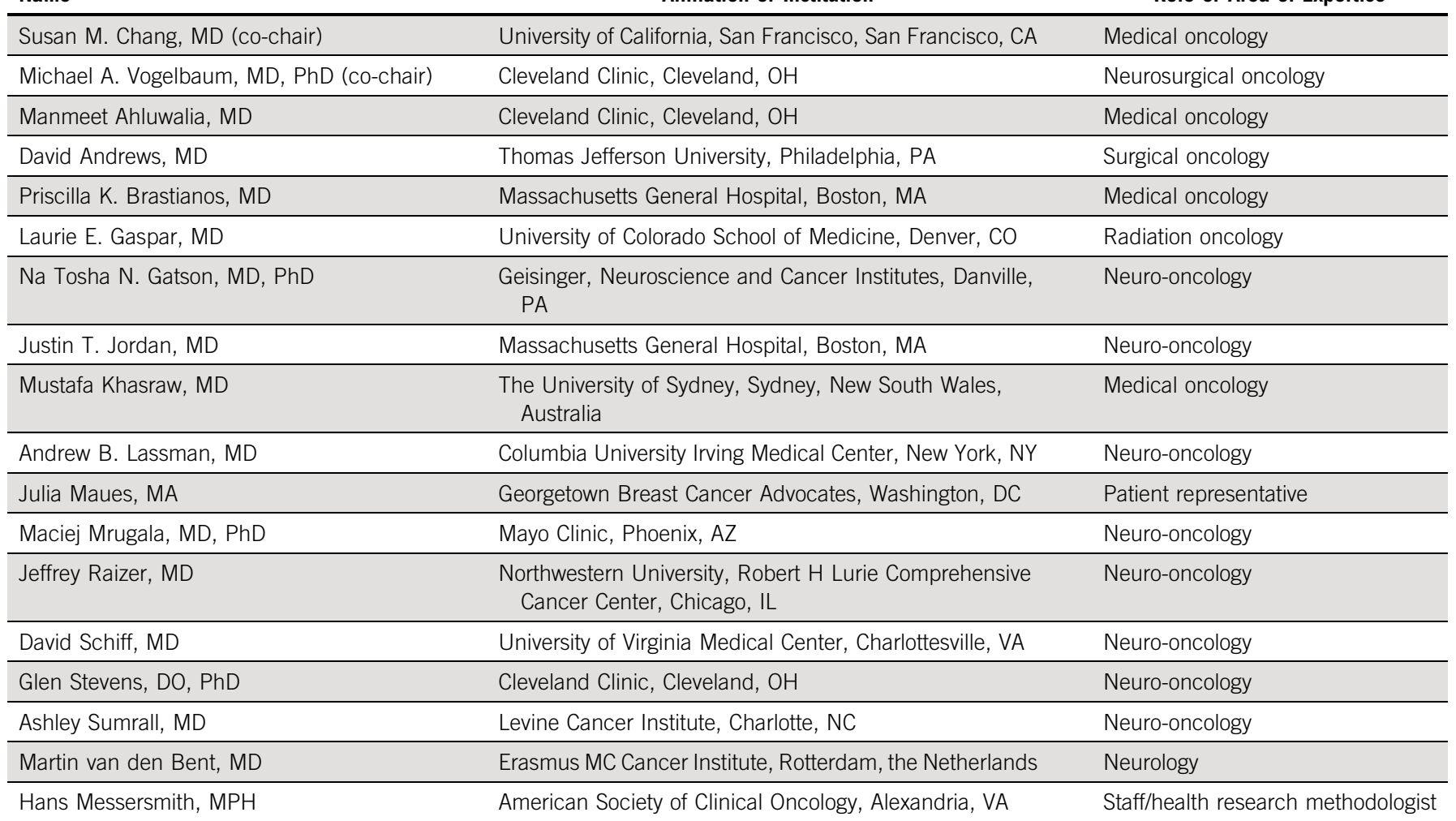

